\title{
The Impact of Mass Media Campaigns on Physical Activity Participation on a Global Scale: Lessons Learned From the COVID-19 Pandemic
}

\author{
Pazit Levinger and Keith D. Hill
}

Physical activity is well known for its health benefits. Despite this, in 2016, more than a quarter (1.4 billion) of the world's adult population were not getting enough physical activity to maintain their health. ${ }^{1}$ Physical inactivity and sedentary behavior increase the risk of many adverse health conditions, morbidity, and mortality, as well as pose a major economic burden worldwide. ${ }^{2}$ The global economic cost for physical inactivity was conservatively estimated at around $\$ 53.8$ billion (INT\$) worldwide for the health care system in $2013 .{ }^{3}$ In addition, physical-inactivity-related deaths contributed to $\$ 13.7$ billion in productivity losses, and physical inactivity was responsible for 13.4 million disability-adjusted lifeyears worldwide. ${ }^{3}$ To tackle the concerning level of physical inactivity worldwide, the World Health Organization released an action plan to promote physical activity, with a goal to reduce physical inactivity by $10 \%$ in 2025 and by $15 \%$ in $2030 .{ }^{4}$ A recent study evaluating the level of physical inactivity across 168 countries reported that, if current trends continue, the 2025 global physical activity target will not be met. ${ }^{1}$ It seems that a more proactive action is needed that targets physical activity as a priority.

In early 2020, the outbreak of coronavirus (COVID-19) created a global health crisis with devastating health, social, and economic impacts. Despite the changes we have experienced, the crisis also presents an opportunity to positively impact creativity and an opportunity to consolidate new physical activity habits. With many restrictions imposed on the public in an attempt to contain the spread of COVID-19, exercise has been one of only a few reasons people were allowed to leave their homes. This is the first time where physical activity has been recognized, promoted, and campaigned on a global scale. Many countries, including Australia and the United Kingdom, have invested in national campaigns on prime-time TV, radio channels, and social media to advocate for the importance of physical activity to maintain people's health during the pandemic. We have never before seen a united action that placed physical activity as part of governments' key messaging and communication. Importantly, these single-branded national media campaigns have been promoted with coordinated support from jurisdictions, state, and local governments. The positive impact of such a campaign is evident in a recent study conducted in England, reporting that two thirds of adults consider exercise to be more important than ever during the current COVID-19 crisis. ${ }^{5}$ The majority $(65 \%)$ of people also believed exercise was helping them with their mental health during the outbreak, with various age groups

Levinger is with the National Ageing Research Institute, and the Institute for Health and Sport (IHES), Victoria University, Melbourne, VIC, Australia. Levinger and Hill are with the Rehabilitation, Ageing and Independent Living (RAIL) Research Centre, Monash University, Melbourne, VIC, Australia. Levinger (p.levinger@nari.edu.au) is corresponding author. reporting to have done more physical activity than the previous week (before the COVID-19 restrictions, compared with a week later; 43\% increase for those aged 16-34 y and 22\% increase for those aged $55 \mathrm{y}$ and older). ${ }^{5}$ The availability of more time due to the reduction in commuting and nonessential activities may also have enabled people to engage in more physical activities at home or outdoors.

Mass media campaigns can be used to communicate public health messages at the population level and have been shown previously to reduce sedentary behavior and influence sexualhealth-related behaviors and treatment-seeking behaviors. ${ }^{6}$ An assessment of campaigns to promote physical activity shows that, while short-term changes can be achieved, sustained effects are difficult to maintain after a campaign's end. ${ }^{7}$ Indeed, mass media campaigns can directly and indirectly produce positive changes in health-related behaviors across large populations. ${ }^{7}$ What makes a particular campaign work might not be entirely clear, but campaigns that run for longer and are more intensive are likely to be more effective. ${ }^{6}$ The usage of mass media campaigns has been recognized previously as one of the "7 best investments for physical activity," as an effective method in raising awareness of physical activity (and sedentary behavior) and influencing social norms. ${ }^{8}$ The current crisis provides a unique unconventional setting, where various "lockdowns" are in place, with the exception of only essential activities: work/education, shopping for essential supplies, exercise, and attending medical appointments or compassionate visits. With ongoing messaging around the importance of maintaining physical health, physical activity is indeed placed as a key priority, which may create behavioral change to adapt to the current situation. There is a need for strategies to sustain the momentum of these improvements in the nature and amount of physical activity beyond the COVID-19 restrictions. Indeed, this positive outcome due to COVID-19 highlights the potential positive impact national campaigns and associated governments' policies can have on public health to increase physical activity on a population level. This also provides an important lesson for public health advocates and state/national governments for the potential long-term benefits of such an investment in the prevention of chronic conditions associated with physical inactivity and the associated health-cost savings.

\section{Acknowledgment}

The authors have no conflict of interest to disclose.

\section{References}

1. Guthold B, Stevens GA, Riley LM, Bull FC. Worldwide trends in insufficient physical activity from 2001 to 2016: a pooled analysis of 
358 population-based surveys with 1.9 million participants. Lancet Glob Health. 2018;6(10):e1077-e1086. PubMed ID: 30193830 doi:10.1016/S2214-109X(18)30357-7

2. Lee IM, Shiroma EJ, Lobelo F, et al. Effect of physical inactivity on major non-communicable diseases worldwide: an analysis of burden of disease and life expectancy. Lancet. 2012;380(9838):219-229. PubMed ID: 22818936 doi:10.1016/S0140-6736(12)61031-9

3. Ding D, Lawson KD, Kolbe-Alexander TL, et al. The economic burden of physical inactivity: a global analysis of major noncommunicable diseases. Lancet. 2016;388(10051):1311-1324. PubMed ID: 27475266 doi:10.1016/S0140-6736(16)30383-X

4. WHO. Global Action Plan on Physical Activity 2018-2030: More Active People for a Healthier World. Geneva, Switzerland: World Health Organization; 2018.
5. Sport England. Survey into adults physical activity attitudes and behaviour. 2020. https://www.comresglobal.com/polls/sport-englandsurvey-into-adult-physical-activity-attitudes-and-behaviour/

6. Stead M, Angus K, Langley T, et al. Mass media to communicate public health messages in six health topic areas: a systematic review and other reviews of the evidence. Public Health Res. 2019;7(8). doi:10.3310/phr07080

7. Wakefield MA, Loken B, Hornik RC. Use of mass media campaigns to change health behaviour. Lancet. 2010;376(9748):1261-1271. PubMed ID: 20933263 doi:10.1016/S0140-6736(10)60809-4

8. Bauman A. Addressing population levels of physical activity requires investment beyond the health sector. Health Promot J Austr. 2018; 29(suppl):10-12. doi:10.1002/hpja.177 